\title{
Impact of fat type (dairy or soy) on postprandial plasma and chylomicron responses with special emphasis on molecular level differences during the postprandial state
}

\author{
M. P. Bonham, K. M. Linderborg, A. Dordevic, A. E. Larsen, J. Weir, P. Gran, P. Meikle, \\ D. Cameron-Smith, M. K. Luotonen, H. P. T. Kallio and A. J. Sinclair \\ School of Exercise and Nutrition Sciences, Deakin University, Australia (MB, AD, AL, PG, DCS), School of Medicine, \\ Deakin University, Australia (AJS), Baker Heart Research Institute, Melbourne, Victoria, Australia (PM, JW), and \\ Department of Biochemistry and Food Chemistry, University of Turku, Finland (KL, HK, ML)
}

Postprandial hyper-triglyceridaemia and delayed chylomicron (CM) clearance have been implicated as independent risk factors for CVD. Subjects diagnosed with metabolic syndrome (MetS) have impaired lipid metabolism as well as an exaggerated postprandial response following dietary intervention. Milk fat contains short and medium chain fatty acids and is one of the most complex dietary fat sources. The aim of this investigation was to examine the postprandial plasma and CM TAG concentrations and TAG molecular species responses to high fat feeding (dairy and soy) from people identified with MetS compared with healthy subjects. Special emphasis was laid on molecular level differences in CM lipids during the postprandial state. A randomised cross-over trial was conducted in a total of twenty-one male subjects (aged 40-60 years). Plasma and chylomicron TAG responses to breakfast meals containing dairy fat or soy oil as well as plasma glucose and concentrations were followed for $5 \mathrm{~h}$. The molecular species of TAG and selected regioisomers from CM were measured by high pressure liquid chromatography-MS/MS, and fatty acids by GC.

Postprandial plasma glucose and insulin responses were significantly elevated in the MetS subjects following high fat breakfast meals. There was no effect of breakfast type on postprandial plasma TAG levels in control or MetS subjects, but postprandial CM TAG concentrations were significantly reduced in all subjects fed the dairy breakfast relative to the soy breakfast at $3 \mathrm{~h}$. Subjects with MetS did not have an altered postprandial CM TAG response compared with healthy control subjects. LC-MS analyses of CM TAG indicated that more than a third of the 80 TAG molecular species detected in the dairy breakfast lipids were essentially absent from the CM TAG at $3 \mathrm{~h}$ postprandially. Most of the food TAG not found in the CM were trisaturated TAG (mainly containing short and medium chain fatty acids). Long chain saturated TAG were also lost from the soy breakfast lipids in comparison with soy CM TAG. In CM TAG from both breakfast types, there was substantial evidence of remodelling of the food TAG molecular species into the CM TAG molecular species. Significant differences in chylomicron TAG FA composition, molecular weight species and TAG FA regioisomers were detected between 3 and $5 \mathrm{~h}$ postprandially.

The composition of dietary fat influences postprandial CM TAG levels irrespective of subjects fasting metabolic profile. Preferential positioning of the shorter and medium chain dairy fats in the TAG molecule in dairy foods limits their incorporation into CM TAG contributing to the reduced postprandial CM TAG levels observed in the subjects who consumed the dairy breakfast. It is possible that these shorter and medium chain fatty acids are used for energy either in gut mucosal cells or following transport to the liver. Differences in chylomicron lipids 3 and $5 \mathrm{~h}$ postprandially indicate selective fatty acid and/or monoacylglycerol absorption, formation and/or clearance. The implications of the findings from this acute research study should be explored in longer-term studies.

This project was supported with funding from The Dairy Health and Nutrition Consortium (DHNC). 